INFO580: Research Project Proposal

\title{
Electronic records management at the coal face: Testing a behaviour search model for EDRMS
}




\section{Table of Contents}

\section{Problem Statement}

1.1 Research Rationale..........................................

1.2 Research Objectives.....................................3-4

1.3 Research Questions.........................................4

1.4 Theoretical Framework....................................4-5

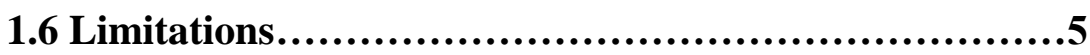

2. Literature Review ........................................5-11

3. Research Design

3.1 Research Sample..............................................11

3.2 Research Methodology........................................11

3.3 Data Collection..................................................12

3.4 Identification of Variables.................................12-13

3.5 Testing Process............................................14

3.6 Data Analysis..............................................14-15

3.7 Ethical Considerations..................................15-16

4. Usefulness of the Research..................................16

5. Research Timeline............................................16

6. References.................................................17-19

\section{List of Appendices}

Appendix A: Gantt chart for INFO580 research tasks and timeline Appendix B: Semi-structured interview schedule 


\section{Problem Statement}

\subsection{Research Rationale}

There is increasing awareness of the dynamic ${ }^{1}$ role of information professionals as more research is applied to the management of electronic information. Ataman (2009) describes this changing role as moving from "people who assist in accessing information" to "people who design and create ways to access information" (p. 217). For successful outcomes, it is not enough to train in the use of an electronic information system if the system is not fit for purpose. The more we understand how and why people use systems and incorporate this learning into system design, then the less effort will be needed to encourage use of the system.

Introducing a new electronic document and record management system (EDRMS) into an organisation consumes a large amount of money and effort which is not effectively spent if the system is inefficient to use (takes up a lot of the participant's time and is cumbersome), or is not used at all. The emphasis is shifting from the records management perspective to the participants. Bailey and Vidyarthi criticise the records management standard ISO15489 (International Organisation for Standardisation, 2002) for emphasising the benefits to the organisation rather than to the participants "Virtually every recommendation is defined in terms of what "the organization" requires and what is in "the organization's" best interests" (2010, p. 281). There is increasing interest in and awareness of how people work and interact with systems and in how systems could support ways of working to better enable effective system implementation and a productive workplace.

\subsection{Research Objectives}

The main objective of this proposed research is to test the reliability and validity of the information search model presented by Joseph, Debowski \& Goldschmidt (2013a) which relates to searching in EDRMS. In order to do this, the search behaviour of employees

\footnotetext{
1 "capable of change while the system continues or program continues to run" The New Zealand Oxford Paperback Dictionary.
} 
using the EDRMS eDOCS Hummingbird in a New Zealand local authority will be compared with the model.

Another research objective is to gain information about current search behaviour in relationship to the design of the new EDRMS system Objective which will soon be installed in the test organisation. As the literature review shows, introducing an EDRMS to a workplace is difficult, requiring a major change to the way that employees have managed their information, (Maguire, 2005). The last objective is to gain insights from the search behaviour in regards to training for the new system.

\subsection{Research Questions}

Research Question 1. How does the search behaviour of individual EDRMS users in the chosen organisation compare to the information search behaviour model of Joseph, Debowski and Goldschmidt (2013a)?

What is the search behaviour of the individual EDRMS users?

Does the search behaviour follow the seven search processes and various steps predicted by the model?

Research Question 2. What does the search behaviour in the current EDRMS indicate for the design of the new EDRMS?

Research Question 3. How has the training affected the search behaviour of users of the current EDRMS?

\subsection{Theoretical Framework}

The framework of the study is designed to test the model. The test situation is, to a large extent, replicating the original research framework but on a smaller scale and in a different setting. One of the original organisations used to develop the model was a local council and the organisation being used to test is a provincial unitary local authority. The unitary status means that a wide range of work is encompassed, including both territorial and regional council roles. This test organisation is situated in another but similar country. 
This research will examine the "microlevel" of the framework employed by Joseph, Debowski and Goldschmidt, that is, "how workers search for information using the EDRMS and how this is aligned to the system" (Joseph, 2010, p. 16).

\subsection{Limitations}

The scope of this study is smaller than the original research to produce the mode. This study tests 10 participants in one organisation against the model whereas the original research tested 40 over four organisations. The questionnaire used has been shortened and slightly tailored for the organisation. The section of the original research dealing with records management principles and theory is not included in this research.

\section{Literature Review}

\section{Information Search Behaviour}

“Information searching can be defined as users' purposive behaviors in finding relevant or useful information in their interactions with information retrieval (IR) systems" (Xie (2011). Xie explains that information searching can also be described as IR, informationseeking and information access. Information seeking, however, takes the larger view of behaviour demonstrated when users are purposefully interacting with information systems to satisfy their goals, whereas information searching refers to the detail of the interaction behaviour with information systems. Xie also determines IR to be a broader concept than information searching, somewhat similar to information seeking but focused on computer-based information systems. The information access concept is the ability to find information, and also to use it.

Timmers and Glas (2010) discuss information seeking behaviour as a "multidimensional construct". As an aspect of information literacy, it encompasses "a wide variety of knowledge, skills and actual behaviour relating, among others (to) localisation, evaluation and effective use of information" (p. 47). The interrelationships between the concepts in information seeking are discussed by Wilson (2006) and information search shown to be the demands made on Information Systems. He also explains that 
"information seeking is not an activity but a set of "actions" that support some higher level activity" (2006b, p.12). Information searching can be seen as one of those actions.

EDRMS are now commonplace in many workplaces as the storage system for corporate information in many formats. An EDRMS, as an Information System ${ }^{2}$, can be operated as a service system and "should be modeled as though the customer's needs and interests genuinely matter" (Alter 2009, p. 201). Alter's research aims to explore what the customers' needs are, as evidenced by their information seeking behaviour.

Information seeking behaviour is relative to the context in which is it displayed (Timmers \& Glas 2012). The information culture of the organisation as part of the cultural context of the organisation's unique "corporate culture" is discussed by Oliver (2007). She explains that, within the organisational context, the construction of information culture is through values given to information and attitudes towards it. This is manifest through how records are stored, accessed, retrieved, and the extent of information sharing. Organisational culture, among other factors, can impact on the use of the system: "studies ... have shown that even systems that are very good technologically do not work, or are not being used as intended, if they do not fit the culture of the organization, or if they are incorrectly implemented, especially without good and proper training" (Gunnlaugsdottir, 2009, p. 61).

As early as 1984, Ellis was intent on developing an information seeking behaviour model "that could inform the development of information retrieval systems" (Ellis, 2005, p138). The literature search shows that, for almost a decade, there has been awareness of the impact of the implementation of EDRMS on the workplace, and reflection on this in terms of lessons learnt. Models of information search behaviour have been developed, but not specifically related to EDRMS. For instance, Xie's definitive article dated 2011 outlines four major digital environments for information searching and search models but does not include EDRMS.

\footnotetext{
2 "An information system is a work system whose processes and activities are devoted to processing information, i.e., capturing, transmitting, storing, retrieving, manipulating, and displaying information. (Alter, 2009, p. 202).
} 
In recognising and attempting to fill this space, Joseph, Debowski and Goldschmidt (2013a) chose to test the information seeking behaviour models described by Ellis (1989), Meho and Tibbo (2003) and Marchionini (1995) as these had been applied to a range of disciplines and "are widely cited as best describing the ways users search for information" (p. 2). Forty EDRMS users from four workplaces were involved in the research. There were differences in the participants' reasons for seeking information as well as in "user population, information sources and search foci" (p.8). The common denominator was the focus on "information need, information gap and/or anomalous state of knowledge" (Belkin, 1980; Dervin, 1992; Wilson, 2005, as cited in Joseph, Debowski \& Goldschmidt, p.8). Within these different contexts, the research tested whether the models were comparable and resulted in a modified information search model for EDRMS. The modifications relate to the moves after EDRMS users stop their search and the behaviour which differentiates simple and difficult searches.

The model under review is basically a flow chart of the search process detailing seven search stages and tactics and moves within those. Xie (2011) explains that "Search models are illustrations of patterns of information searching and the search process" The search process is dynamic and it is the variety of variables that define the process.

Some findings from the literature review point to IT/technology systems as engagement catalysts in the workplace, emphasising staff participation rather than staff as end-users or customers (Alter 2009; Bailey \& Vidyarthi 2010). As familiarity and confidence with EDRMS in the workplace grows, it could be predicted that participants will expect and even demand that electronic systems meet their as well as corporate needs, if these are seen to be at odds.

The implementation of an EDRMS involves this interaction between the business and technology and presents an "interdisciplinary design issue." It is an environment where "creators and users need to work in partnership to ensure the ongoing usability of records" (Wilkins, Swatman \& Holt, 2009, p. 39). The landscape is complex when the regulatory side of records management, standards and statutory requirements is added. 
As well, EDRMS is an enterprise-wide system (EWS) and requires effective widespread dissemination across the organisation and integration with other systems.

\section{EDRMS design}

"When work system participants in the same roles have significantly different capabilities and interests, the design of the system may have to accommodate those differences" (Alter, 2006, p. 69). Alter questions "How well are the roles, knowledge, and interests of work system participants matched to the work system's design and goals?" (Alter, p. 25).

Bailey (2010, p. 280) also asks the question "how well do we truly understand not just how our users work, but why they work the way they do?" Implementations of EDRMS are not enthusiastically welcomed in the workplace as they involve changes to practice which are often seen as more trouble than good. Systems need to be fit for purpose - but what or whose purpose is that? Setting up a system that purely fulfills recordkeeping requirements will have limited success if it does not also meet the participants' needs. "The success of the software should be evaluated in terms of how well it helps people do their work, not in terms of its theoretical capabilities or how well it operates on a computer" (Alter, 2006, p. 217).

Alter (2006) believes that systems in organisations are best understood as "work systems in which human participants and/or machines, perform work using information, technology, and other resources to produce products and/or services for internal or external customers" ( $\mathrm{p}$ 11). The design and goals of the system should be matched to the participants' roles, knowledge and interests.

This is a step forward from the views expressed by McLeod, Hare and Johare (2004) who state that as a priority the system design must guarantee appropriate creation and capture, therefore providing reliable evidence of and information about the business transactions. While this is patently true, there is no mention of the participants' needs. Further, the authors state that learning about new responsibilities for recordkeeping is uppermost for “empowered users" (p. 5). 
Singh, Klobas and Anderson (2008, p. 53) instead, recommend that a separate "user friendly classification scheme" that is "...intuitive and aligned to the users' work processes and thinking patterns" be developed in parallel with the formal records management classification scheme and would live in the background for retention and disposal purposes. This set-up would alleviate the problems that users have with learning the complexities of records management tools.

Maguire's survey (2005) showed that the benefits of the new EDRMS implemented in a the Estates Department of the British Library, such as the ability to document share, did not offset the dissatisfaction of the staff with aspects of the system design and capability, for example, the lack of drag and drop functionality, the necessity for metadata entry, and the confusing thesaurus indexing. The outcome of this dissatisfaction was that the system was not well adopted. Overall, the system needs to be seen as an improvement for participants rather than an extra burden and Maguire states, "in spite of extensive training, most staff never got to grips with the system" (Maguire, p.150).

\section{Training}

Gunnlaugsdottir (2009) does make a correlation between training and the increased use of electronic records management systems (ERM). The estimated use in one of the studied organisations rose from 15 to 50 percent after an increased training effort in both general records management knowledge and individual training. The question arises - is the lack of use due to the system not being user-friendly or simply the lack of effective training? She refers to an Icelandic saying, "those lacking in the skills to row a boat may excuse their inability by placing the blame on the poor design of the oars" (p. 70).

Norton, Coulson-Thomas, Coulson-Thomas and Ashurst (2012) are of the opinion that the problem of not realising the benefits of a new system, that is, technical-isomorphism (getting the system up and going but not achieving competitive advantage) and system atrophy ( failure to maintain the system) can be alleviated by end-user and postimplementation training. They put forward nine recommendations for training for highly demanding information systems (HDIS). An EDRMS could be included in this category 
as HDIS are defined as "configurable information systems packages that integrate information and information based processes within and across functional areas in an organisation" (p. 647).

The authors come close to exploring the user perspective in their discussion of system specifics "showing different ways the client can do things" (Norton, Coulson-Thomas, Coulson-Thomas and Ashurst, 2012, p. 651), and also in the mapping of business processes to suit the client and then delivering bespoke training specific to each customer. They recommend that more resources be committed to training and believe that communicating the benefits of the system to the recipients is "absolutely critical" (p.653).

Bailey and Vidyarthi (2010) assert that there would be no need to sell the benefits if the system made everyday working life easier rather than harder. They take a bottom up view of records management, with the primacy of users needs over those of the organisation. The possibilities of the developing field of human computer interaction (HCI) which "researches human behaviour in the context of technology, and uses the results to design effective system" (p. 284) are explored in order to develop a system that users would naturally want to interact with. The objective of standardisation in records management could seen to be counter to developing user-friendly systems, however, the authors' answer is to be able to provide personalised views of the system which enhance the users' control.

Personalisation of training, for McLeod, Hare and Johare (2004), means tailoring to individual needs around participants' role and their understanding of records management. Accordingly training should be a "combination of horizontal and vertical mappings..." (p. 7) which includes a macro level view and a micro level exploration, as well as being informed by the organisation's business processes. This is similar to the approach recommended by the standard (ISO 15489:1, 2001) in that organisations should "establish an ongoing programme of records training..." which is based on roles and responsibilities. 
"Records management is heavily reliant on the participation of individual and collective users to achieve its aims and yet understanding of their requirements is often basic and simplistic" (Bailey \& Vidyarthi, 2010, p. 279). Joseph, Debowski and Goldschmidt (2013a) have contributed to understanding the search behaviour of EDRMS users, concluding that the EDRMS design, the specific search task, and the training received, all impacted on search behaviour. If re-design of the system to suit participant's needs, as proposed by Bailey \& Vidyarthi (2010), is not practicable, then training in the search options provided by the system design is the only option.

\section{Research Design}

The design of this qualitative research closely follows that explained by Joseph, Debowski and Goldschmidt (2013b) to produce the model that is being tested. The scope of this study was discussed with Pauline Joseph (personal communication, April 18, 2013) along with some basic design features. She is supportive of research testing the model.

\subsection{Research Sample}

A selection of staff will be invited to participate. The selected staff must be using the current EDRMS and will be selected to represent a cross section of the organisation. The sample size will be 10 from a total population base of 274FTEs, of which 235 use the EDRMS.

\subsection{Research Methodology}

The research method is the same as the method used to construct the model described by Joseph as qualitative using a "constructivist research paradigm and perspective" (Joseph, 2010, p. 116). Although this research is undertaking the quantitative role of testing a model, the general approach to data collection is qualitative as described by Bryman (2008) as "the stress is on the understanding of the social role through an examination of the interpretation of that world by its participants." (p. 366). 


\subsection{Data collection procedures}

The framework for the collection of the data is structured in the same way as the data collection for the construction of the model, which is, based around questionnaires and protocol analysis.

A short structured questionnaire will be used to collect background data about the participants; a semi-structured questionnaire of 20 questions targeting search behaviour (this questionnaire is a shortened version of that used to develop the model); and protocol analysis. The specific protocol analysis method that will be used is that of "concurrent verbal reports" (Ericcson \& Simon, 1993, as cited in Austin \& Delaney, 1998, p.42). The protocol analysis will ask the participants to recall the most recent simple and difficult search they conducted using the EDRMS, and then to demonstrate these searches while verbalising their strategies.

The background data will be gathered before the semi-structured search behaviour questionnaire is conducted in a face-to-face interview situation. The protocol analysis will follow directly after and will take place at the participant's work station. Permission will be sought from participants to make a sound recording of the interview and the "talk aloud" aspect when the participants demonstrate their search method. This will allow for transcription.

The range of data collection methods used in this research enable the information provided to be checked for reliability; for example, the way participants describe their search behaviour may be different to their demonstration of it.

The EDRMS used for this research is eDOCs Hummingbird v 3.7, which was installed in the organisation in August 2007 and was the first EDRMS for the organisation.

\subsection{Identification of Variables}

Specific background data, gender, age, work role and time worked in the role, will be gathered from the research participants and will be analysed. 
From the Research Questions, five variables were identified.

\begin{tabular}{|l|l|}
\hline Variables measured & $\begin{array}{l}\text { Research Questions } \\
\text { RQ1 How does the search behaviour of } \\
\text { individual EDRMS users compare to the } \\
\text { information search behaviour model of } \\
\text { Joseph, Debowski \& Goldschmidt? } \\
\text { 1. The individual search behaviour of 10 } \\
\text { EDRMS users. } \\
\begin{array}{l}\text { 2. Search behaviour of the 10 EDRMS } \\
\text { users compared with the model. }\end{array}\end{array}$ \\
$\begin{array}{l}\text { the individual EDRMS use? } \\
\text { 3. Design differences between current and } \\
\text { new EDRMS. }\end{array}$ & $\begin{array}{l}\text { RQ2 the search behaviour follow } \\
\text { the seven search processes and } \\
\text { various steps predicted by the } \\
\text { model? }\end{array}$ \\
\hline $\begin{array}{l}\text { 4. Training delivered by the organisation. } \\
\text { 5. Training received by the 10 individual } \\
\text { EDRMS users. }\end{array}$ & $\begin{array}{l}\text { RQ3 How has training affected the search } \\
\text { behaviour of users of the current EDRMS? }\end{array}$ \\
\hline
\end{tabular}

\section{Table 1: Five variables related to Research Questions}

The process to measure these five variables will be as described below in the Data Analysis section.

The training that participants have received is another variable which may impact on search behaviour. As discussed earlier, Joseph, Debowski and Goldschmidt (2013a) found that training did have an impact on search behaviour, as did the EDRMS design and the search task. 


\subsection{Testing Process}

Searching is a dynamic process and made up of a variety of variables which will be tested for validity and reliability in this specific context. The triangulated data from each research participant, that is, the questionnaire and the simple and difficult searches, will be flowcharted for comparison and amalgamated into one search strategy per participant. This outcome will be compared with the search strategies of the other research participants to produce one flowchart of search behaviour which can be compared with the model.

A test run of the questionnaires, interview and the protocol analysis will be done for validity within this context and also to gauge the time needed for completion so that participants can be accurately advised of their time commitment. The coding schedule will be tested to determine if the correct options have been identified.

\subsection{Data Analysis}

To answer the research questions, the data will be analysed using Microsoft Excel. The analysis will involve;

- RQ1, identifying the search behaviour of the 10 participants from the data gathered from the individual interviews (questions 3-15 and 20) and the protocol analysis for the simple and difficult searches which will enable the search patterns to be produced and then be compared with the seven search processes and various steps of the model. Any differences between the participants' description of their search and the search itself will be discussed at the time of the search demonstration. The protocol analysis data is limited to the users' most recent simple or difficult searches. This analysis is identical to that used to construct the model as explained in Singh, Klobas and Anderson (2008).

- $\mathrm{RQ} 2$, the design of the current EDRMS will explained and compared to the proposed design of the new EDRMS and analysed in relationship to the search behaviour evidenced from RQ1.

- $\quad \mathrm{RQ3}$, data compiled from the questionnaire (questions 16-19) will be compared with information collected about the current EDRMS training programme of the 
organisation. This will show the effect of the current training delivered by the organisation on the search behaviour of the participants.

To analyse the data, the responses to the semi-structured questionnaire and the protocol analysis will be coded according to a coding schedule. As far as possible, the coding will be that used by Joseph (2010) but may need to be contextualised if, as sample data is analysed, significance was not accounted for. The participants' background data, the current training programme information, and the data from the semi-structured interviews will be linked along with the protocol analysis.

\subsection{Ethical considerations}

As this research involves people, it needs to be approved by the Victoria University's School of Information Management Human Ethics Committee (HEC) before data collection can start. The original research, the outcome of which is being tested by this research, was approved by the University of Western Australia's Faculty of Human Research Ethics Sub-Committee.

An information sheet will be provided to prospective participants making clear, in plain natural language, the purpose of the research and the kinds of information that will be collected. There will be a clear statement about the storage, access to and usage of the data, including timeline for storage. It will be stressed that individuals will not be identifiable, reassuring the participants that their privacy will be protected. The interviews will be recorded, transcribed and stored on the researcher's computer, or if printed will be stored in a locked cabinet accessible only to the researcher. Data protection will involve the use of secure password protected electronic files for data storage and analysis. No personal names will be associated with the data and the presentation of findings will take into account the possible identification of participants, ensuring confidentiality. It will be clearly stated that participation is voluntary and participants will have the opportunity to ask questions and withdraw at any stage, including withdrawal of data supplied. 
The information sheet will include that the HEC has ethically approved the research, and that acceptance of the invitation to attend an interview implies consent. There will be sufficient information provided on the sheet for participants to decide whether or not they wish to participate, that is, to give their informed consent.

\section{Usefulness of the Research}

By testing this model and understanding how staff search the EDRMS, insights will also be gained to inform future design of the system and effective methods for training. The usefulness of the research will therefore be twofold:

- Providing further information about the validity and reliability of the information search model

- And to assist the organisation with an effective implementation by understanding how the design of the new EDRMS matches the search behaviour of staff and how training can be tailored to fit staff needs.

\section{Research Timeline}

See Gantt chart in Appendix A for timetable. 


\section{References}

Alter, S. (2009). Viewing systems as services: A fresh approach in the IS field.

Communications of the Association for Information Systems, 26 article 11, 195224.

Alter, W. (2006). The work system method: Connecting people, processes and IT for business results. Larkspur, California: Work System Press.

Ataman, K. K. (2009). Requirements for information professionals in a digital environment: Some thoughts. Program: Electronic Library and Information Systems. 43(2), 215-228.

Austin, J. \& Delaney, P.F. (1998). Protocol analysis as a tool for behavior analysis. The Analysis of Verbal Behavior, 15, 41-56. Retrieved from http://www.ncbi.nlm.nih.gov/pmc/articles/PMC2748634/pdf/anverbbehav000300042.pdf

Bailey, S., \& Vidyarthi, J. (2010). Human-computer interaction: the missing piece of the records management puzzle? Records Management Journal, 20(3), 279-290. Retrieved from Emerald database.

Bryman, A. (2008). Social research methods ( $3^{\text {rd }}$ ed.). Oxford, England: Oxford University Press.

Ellis, D (1989). A behavioral approach to information retrieval system design. Journal of Documentation, 45(3), 171-212. Retrieved from the Emerald database.

Ellis, D. (2005). Ellis's model of information-seeking behavior. In Fisher, K.E., Erdelez, S. \& McKechnie, L. (Eds). Theories of information behaviour. Medford, N. J.: Information Today. Pp 138-142,

Gunnlaugsdottir, J. (2009). The human side of EDRMS: An Icelandic study. Records Management Journal, 19(1), 54-72. Retrieved from the Emerald database.

International Organization for Standardization. (2002). ISO 15489-1: Information and Documentation - records management - Part 1 -General. Geneva, Switzerland: International Organization for Standardization.

Joseph, P. (2010). EDRMS search behaviour: Implications for records management principles and practices. Unpublished doctoral dissertation, University of Western Australia School of Business, Crawley, Australia. 
Joseph, P., Debowki, S., \& Goldschmidt, P. (2013a). Models of information search: A Comparative analysis. Information Research, 18(1), paper 562. Retrieved from http://informationr.net/ir/18-1/paper562.html

Joseph, P., Debowski, S., \& Goldschmidt. (2012). Paradigm shifts in recordkeeping responsibilities: Implications for ISO 15489's implementation. Records Management Journal, 22(1), 57-75. Retrieved from the Emerald database.

Joseph, P., Debowski, S., \& Goldschmidt, P. ( 2013b). Search behaviour in electronic document and records management systems: An exploratory investigation and model. Information Research, 18 (1), paper 572. Available at http://InformationR.net/ir/18-1/paper572.html

McLeod, J., Hare, C., \& Johare, R. (2004). Education and training for records management in the electronic environment: The (re)search for an appropriate model. Information research, 9(3), paper 179. Retrieved from http://informationr.net.ir.9-3/paper179.html

Maguire, R. (2005). Lessons learned from implementing an electronic records management system. Records Management Journal, 15(3), 150-157. Retrieved from the Emerald database.

Marchionini, G. (1995). Information seeking in electronic environments. Cambridge, United Kingdom: Cambridge University Press.

Meho, L.I., \& Tibbo, H.R. (2003). Modeling the information-seeking behavior of social scientists: Ellis's study revisited. Journal of the American Society for Information Science and Technology, 54(6), 570-587. Retrieved from http://onlinelibrary.wiley.com/doi/10.1002/asi.10244/full

Norton, A. L., Coulson-Thomas, Y.M., \& Coulson-Thomas, C. J. ( 2012). Delivering training for highly demanding information systems. European Journal of Training and Development, 36(6), 646-662.

Oliver, G. (2007). Implementing international standards: First, know your organisation. Records Management Journal. 17(2), 82-93. Retrieved from the Emerald database. 
Singh, P., Klobas, J. E., \& Anderson, K. (2008). Information seeking behaviour of electronic document and records management systems (EDRMS) users: Implications for records management practices, part 3. IQ, May, 48-56.

Timmers, C. F., \& Glas, C.A.W. (2010). Developing scales for information-seeking behaviour. Journal of Documentation. 66(1), 46-69.

Wilkins, L., Swatman, P.M.C., \& Holt, D. (2008). Achieved and tangible benefits: Lessons learned from a landmark EDRMS implementation. Records Management Journal, 19(1), 37-53. Retrieved from the Emerald database.

Wilson, T. D. (2006). On user studies and information needs. Journal of Documentation, 62(6), 658-670. Retrieved from Emerald database.

Xie, I. (2011). Information searching and search models In Encylopedia of Library and Information Sciences $\left(3^{\text {rd }}\right.$ ed.). Retrieved from http://www.tandfonline.com.helicon.vuw.ac.nz/doi/full/10.1081/E-ELIS3120043745 


\section{Appendix A.}

Gantt chart for INFO580 research tasks and timeline.

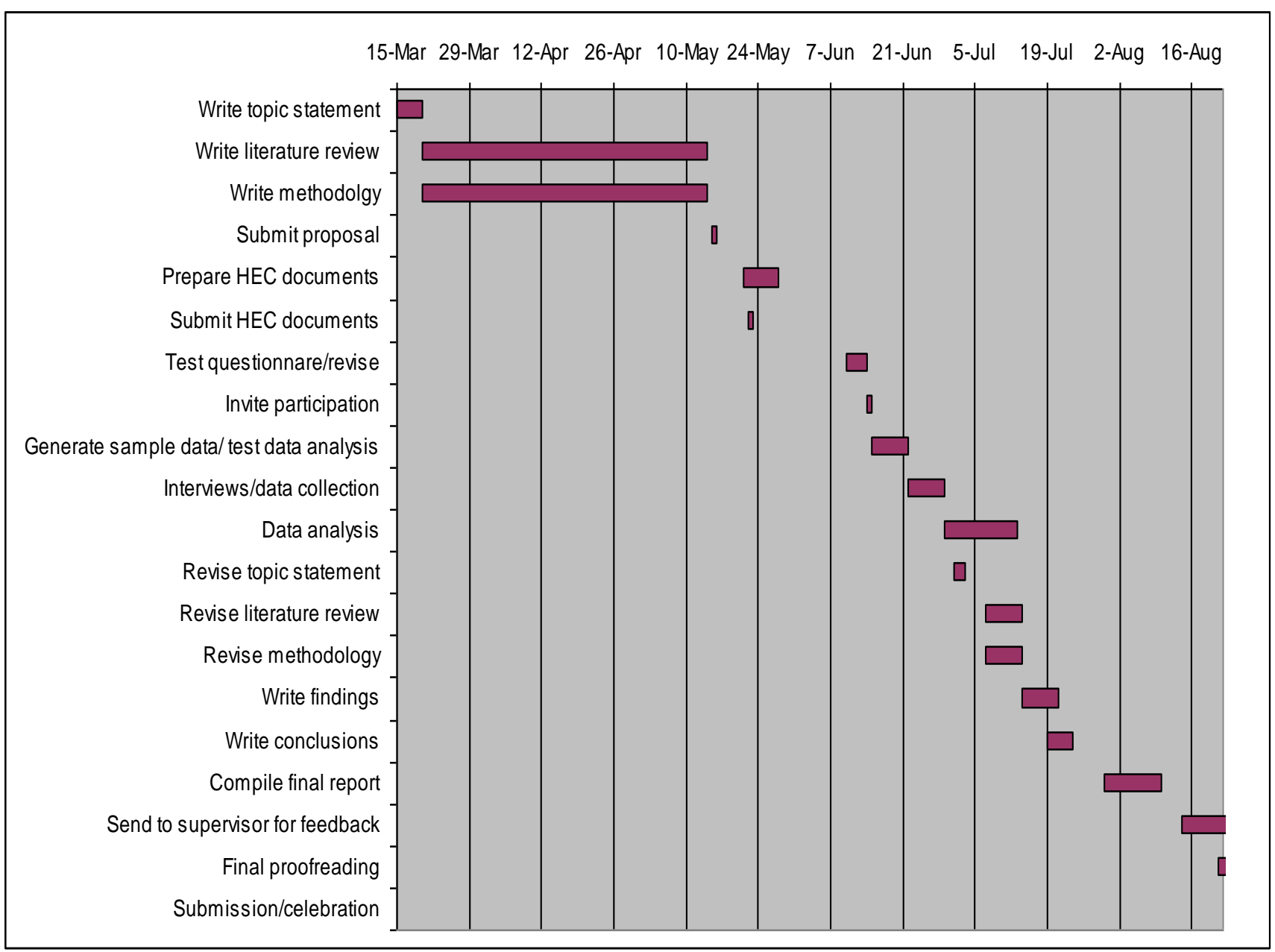




\section{Appendix B}

\section{Semi-structured interview schedule}

Usage:

1. Why do you use the EDRMS?

2. What are the types of information you would search/look for/find in the EDRMS? Probe to find out why they would search for these in the EDRMS instead of other information sources?

\section{Searching patterns in the EDRMS:}

3. Tell me about the different ways you search/look for/find information in the EDRMS?

4. What is your preferred way of searching to FIND information? Probe to find out if they search or browse?

5. If I asked you to describe the registration process to FILE information, how would you describe it? Probe to find out if they find the registration process easy or cumbersome (too much data entry to do?)... any benefit they see in entering all of the metadata?

6. How do you decide when to stop searching further in the EDRMS?

7. Do you "save" your frequently used search criteria?

8. How would you rate your efforts in finding the information you require in the EDRMS? Probe to find out whether it is efficient?

9. How do you keep track of new items added to the EDRMS relevant to your work or projects or of interest to your job function within the EDRMS? Probe to find out how they find this experience - cumbersome, easy, difficult, other comments?

10. What is the most difficult problem you experience in searching for material via the EDRMS?

11. Would you ask for help when searching for information in the EDRMS? If so, when would you ask for help? Who would you ask?

\section{Classification Scheme:}

12. Are you familiar with the classification scheme used in the EDRMS? Can you describe how the classification scheme works in this organisation?

13. Do you use the classification scheme in the EDRMS? If so how? If not why?

14. If I asked you to evaluate the Classification Scheme in the EDRMS, how would you describe it? Probe to find out what they like about the classification scheme and what they would like changed? How many levels should the classification scheme have ie 1 to 2 levels only.

\section{Work Task}

15. How does the work task affect the way that you search? Probe to find out how the search is affected by the type of information sought ie for a general work task and for a council report?

Training

16. Have you had training on the EDRMS?

17. Please describe the training you received.

18. When was the training conducted?

19. If I asked you to evaluate the training you received, how would you describe it?

Design:

20 Explain and show them how their EDRMS is currently designed. Then ask them what do they think of the design of the EDRMS? Probe- what do you like about the design of the EDRMS? Probe - what would you like changed about the design of the EDRMS? 
Student: Heather Mackay

Word count: 4253 (excluding References and Appendices) 